\title{
Design and characterization of conductive biopolymer nanocomposite electrodes for medical applications
}

\author{
Charles TEMATIO ${ }^{1, a}$, Mònica BASSAS-GALIA², Narcis FOSSO ${ }^{1}$, Vanessa \\ GAILLARD², Marc MATHIEU², Manfred ZINN², Enrico STADERINI ${ }^{3}$, and \\ Silvia SCHINTKE ${ }^{1, b, *}$
}

\author{
${ }^{1}$ Laboratory of Applied NanoSciences (COMATEC-LANS), HEIG-VD, University of Applied \\ Sciences Western Switzerland, Avenue des Sports 20, CH-1401 Yverdon-les-Bains, Switzerland \\ ${ }^{2}$ Institute of Life Technologies, HES-SO Valais, University of Applied Sciences Western \\ Switzerland, Route du Rawyl 64, CH-1950 Sion, Switzerland \\ ${ }^{3}$ Institut d'Automatisation Industrielle - Applications Biomédicales, HEIG-VD, University of Applied \\ Sciences Western Switzerland, Route de Cheseaux 1, CH-1401 Yverdon-les-Bains, Switzerland \\ acharles.tematio@heig-vd.ch, ${ }^{b, *}$ silvia.schintke@heig-vd.ch
}

Keywords: nanocomposites, nanowires, biopolymers, electrical conductivity, impedance spectroscopy, medical engineering, electrocardiography

\begin{abstract}
Metal-based electrodes, despite being the most widely used for biomedical applications, are limited by a poor reliable skin-surface interface and patients suffer from comfort issues. The most common problems/inconveniences are caused by stiff electrodes, skin irritation, allergic reaction or corrosion. In order to overcome these problems, we produced and tested flexible electrodes involving biopolymer nanocomposite materials. Conductive polymers have been intensively studied and applied in the field of organic photovoltaics and flexible organic electronics. Recently, the use of conductive biopolymer nanocomposite has also emerged as an interesting and promising material for biomedical applications.

In this study, we have designed and characterized electrodes made of a flexible and conductive nanocomposite material using a biocompatible and biodegradable polymeric matrix of poly(3hydroxyalkanoate) (PHA, in particular poly(3-hydroxybutyrate), PHB) containing conductive nanowires. The biopolymer nanocomposites and their electrical conductivities were investigated by optical microscopy, scanning electron microscopy (SEM) and electrical four-point probing. The electrical conductivities obtained in the different PHA-polymer nanocomposites containing different concentrations of conductive additives is discussed in relation to the nanocomposite structure at the microscopic level. Finally, our developed biopolymer nanocomposite prototype electrodes have successfully been tested for transcutaneous electrical nerve stimulation (TENS) and electrocardiography ECG applications in comparison to conventional electrodes.
\end{abstract}

\section{Introduction}

For many years, biopolymers have been widely used in medicine as substitutes for natural tissues due to their biodegradability, biocompatibility, and thermoplastic properties [1,2]. Some of the most commonly used polymers in biomedical application are polylactide, polyglycolide and more recently polyhydroxyalkanoates (PHAs) [1,3]. PHAs are bacterial polyesters that are synthetized by a large number of microorganisms under specific cultivation conditions. PHAs are accumulated intracellularly as carbon and energy storage compound [4] and based on the number of carbon atoms in the monomer unit, these polymers can be classified mainly into two groups: short-chain-length (scl) PHA and medium-chain-length (mcl) PHA. Scl-PHAs (e.g., poly(3-hydroxybutyrate), PHB) are crystalline and have a tensile strength similar to polypropylene (40 MPa) although being more brittle. Alternatively, mcl-PHAs are amorphous or semi-crystalline elastomers with low melting temperature 
$\left(\mathrm{T}_{\mathrm{m}}<50^{\circ} \mathrm{C}\right)$ and weak tensile strength. Unfortunately, these types of polymers are not conductive per $s e$, therefore additional conductive additives or chemical modification [5,6] are required.

Today, the most widely used medical electrodes for TENS and ECG applications are made of a piece of conductive metal (or printed metal) covered with a hydrogel. Many of these electrodes on the market are reported to be hazardous for the skin under intensive and long term use revealing thus the need of better performing and innocuous medical electrodes [7].

In this paper, we investigated PHA as a base material for conductive nanocomposite electrodes, motivated by the fact that PHAs can be sufficiently rigid [8] and sustainable to bear conductive materials such as nanowires, while preserving thermoplastic properties [9]. In particular, we investigated PHB (scl-PHA) using silver nanowires as conductive additives in order to produce and study conductive nanocomposite membranes, which we study from the material point of view. Furthermore the manufactured prototypes and their potential application as medical electrodes for TENS and ECG are discussed.

\section{Materials \& methods}

Chemicals. The polymer used in this study (PHB) was kindly supplied by Professor Zinn's lab (Institute of Life Technologies, HES-SO Valais Wallis) [10]. All general chemicals and reagents were purchased from Reactolab SA and Sigma-Aldrich.

Nanocomposite film preparation, nanocomposite and electrode characterization. PHA polymers are non-conductive in their native form. Therefore silver nanowires (AgNW) (Blue Nano Inc., USA) had to be blended in. In order to produce nanocomposite thin films, polymeric nanocomposite dispersions were prepared as follows: The polymer solution $(2 \mathrm{wt} \%$ in dichloromethane) was properly mixed with the nanowire suspension at different AgNW/polymer ratios (1-15\% wt of polymer) and thoroughly stirred assuring good homogeneity of the nanocomposite blend. Throughout this paper all AgNW load concentrations are given in wt $\%$ of AgNW content with respect to the polymer content. Polymer nanocomposite films were then solvent casted on glass substrates $(2.5 \mathrm{~cm} \times 2.5 \mathrm{~cm})$ and peeled off upon drying at room temperature at ambient pressure.

The polymer nanocomposite film thickness was determined optically from cross-section images of the nanocomposite membranes using a reversed microscope (Leica DMILM).

SEM images were acquired on a field emission scanning electron microscope ZEISS LEO 1525 using the Everhart Thornley and Inlens SE detector (Carl Zeiss, Oberkochen, Germany) at an acceleration voltage of $10 \mathrm{kV}$. Images were recorded using the Inca EDS/EDX software (Oxford Instruments Tubney Wodds, UK).

Electrical characterization was performed applying the Van-der-Paw four-point collinear probe measurement technique with a lateral tip spacing of $1 \mathrm{~mm}$ and using a KEITHLEY 2400 source meter. The resistivity (for thin films) is given by Eq. 1 [11]:

$$
\rho=d \cdot \frac{\pi}{\ln 2} \cdot R=d \cdot 4.532 \cdot \frac{U}{I}=d \cdot R_{s q}
$$

where $U=$ measured voltage, $I=$ source current, $d=$ sample thickness, $R=$ resistance, and $R_{s q}=$ sheet resistance. The conductivity $\sigma$ is given as the inverse of the resistivity $\rho$ as determined from Eq. 1. The values were determined for driving currents ranging from $1 \mathrm{~mA}$ to $100 \mathrm{~mA}$. The presented conductivity values for each composite material and for each AgNW load concentration are based on measurements performed on at least three samples, where each sample was characterized for at least 9 different positions.

Electrode device characterization. Nanocomposites were tested in comparison to commercial electrodes for medical applications (TENS and ECG). Tests for TENS applications were performed using a Tesmed TE-670 digital TENS apparatus, whereas ECG signal acquisitions were performed using a Ti ADS 1298ECG-EEG-FE system. A Bode 100 impedance meter was used for impedance spectroscopy measurements of the medical electrode devices. 


\section{Results and discussion}

Nanocomposite conductivity and microstructure. Conductivity and thickness of PHB films for various $\mathrm{AgNW}$ load concentrations (1 wt $\%$ to $15 \mathrm{wt} \%$ ) are shown in Figure 1. As an overall feature, we observe as expected that the conductivity increases with increasing AgNW concentration, reaching a value of $(6.11 \pm 0.04) \cdot 10^{4} \mathrm{~S} / \mathrm{m}$ at $15 \mathrm{wt} \% \mathrm{AgNW}$ concentration. However, at $5 \mathrm{wt} \%$ concentration, a drop in the conductivity is observed (Fig. 1a), although the film thickness gradually decreases (Fig. 1b). In order to assess the homogeneity and the distribution of conductive additives within the nanocomposite films as a function of the AgNW concentration in more detail, SEM measurements were performed (Fig. 2).

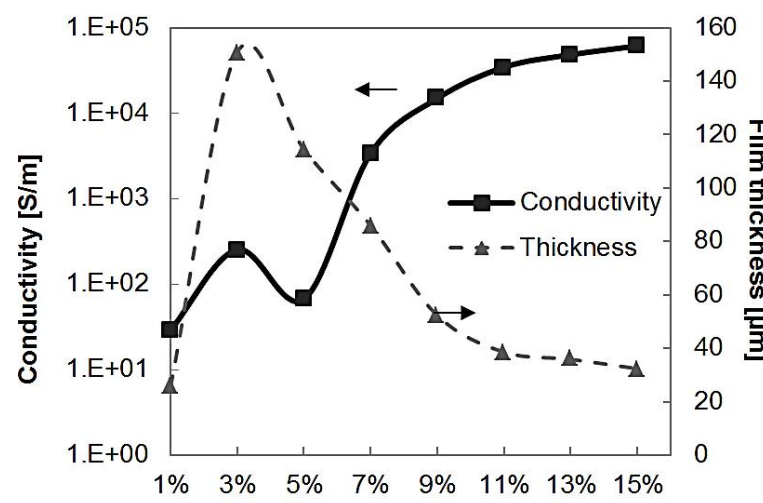

a)

AgNW load concentration [wt\%]

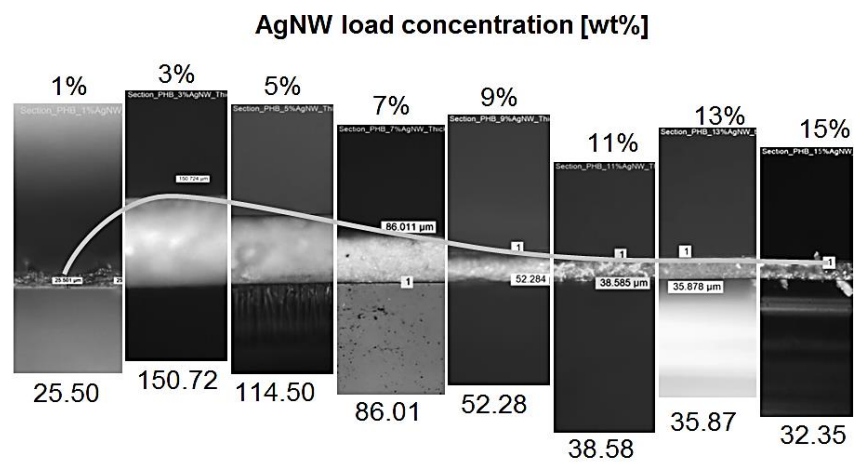

b)

Thickness [ $\mu \mathrm{m}]$

Fig.1: PHB nanocomposites: a) surface conductivity and film thickness as a function of AgNW load concentration; b) optical micrographs of PHB nanocomposite films (cross-sections) for various AgNW load concentrations (1 wt $\%$ to 15 $\mathrm{wt} \%)$.

At $3 \mathrm{wt} \%$ AgNW concentration, SEM images reveal the formation of a porous scaffold structure with pore diameters significantly larger than the walls of the scaffold structure (Fig. 2). This observed porous microstructure formation, partially initiated at an $\mathrm{AgNW}$ concentration of $1 \mathrm{wt} \%$ (Fig. 2), is consistent with a significant increase of the film thickness observed at $3 \mathrm{wt} \%$ concentration (Fig. 1).
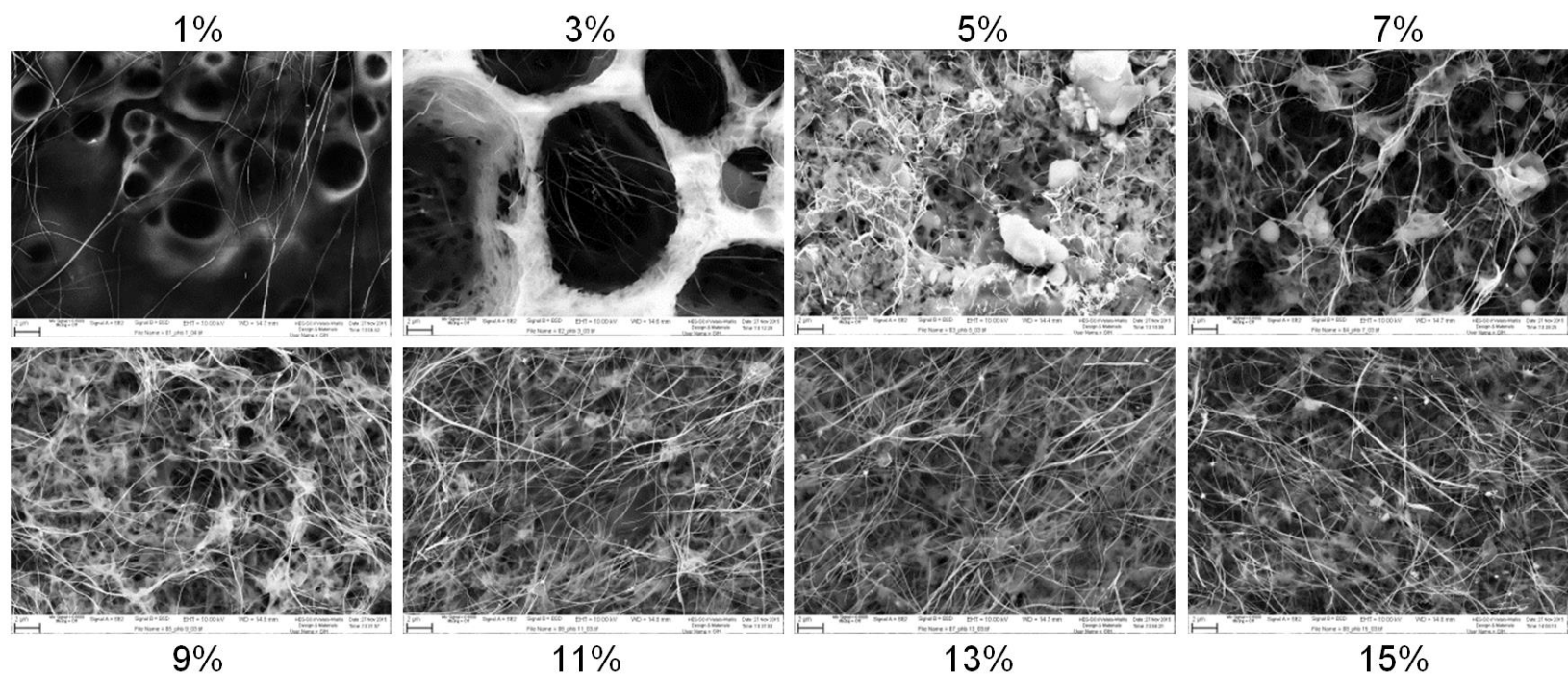

Fig.2: SEM micrographs of PHB nanocomposite films with increasing AgNW concentrations from $1 \mathrm{wt} \%$ to $15 \mathrm{wt} \%$, (scale bar $2 \mu \mathrm{m}$ ).

The film thickness decreases for nanowire concentrations higher than $3 \mathrm{wt} \%$, (Fig. 1b), which is consistent with the structural changes observed in SEM images (Fig. 2): in case of AgNW concentrations higher than $5 \mathrm{wt} \%$, significantly smaller pores are observed and especially in the case 
of AgNW concentrations of 11-15 wt \% the formation of homogenous networks of AgNWs can be observed.

We therefore attribute the high conductivity at $3 \mathrm{wt} \%$ load concentration to the dense packing of the AgNWs observed within the porous scaffold walls while only a few nanowires are observed within the pores (Fig. 2b). The drop in conductivity observed at $5 \mathrm{wt} \%$ (Fig. 1a) is consequently attributed to a microstructural transition of the nanocomposite films from a porous nanocomposite scaffold structure to a homogeneous nanowire network structure.

For our further investigation and medical electrode design we chose thus in particular the PHB with $3 \mathrm{wt} \% \mathrm{AgNW}$ load concentration as a promising nanocomposite material, as its conductivity of $(2.53 \pm 0.02) \cdot 10^{2} \mathrm{~S} / \mathrm{m}$ is expected to be sufficiently high for electrode applications and requires only a low concentration of $\mathrm{AgNW}$.

Prototype design and testing. We designed prototypes of nanocomposite based on medical electrodes by replacing the hard metallic electrode part of commercial models of medical electrodes (gel interface electrodes) using the nanocomposites PHB $+3 \mathrm{wt} \% \mathrm{AgNW}$. The demonstrator nanocomposite electrodes were tested for both, nerve stimulation and electrical signal acquisition. Our prototypes were tested in comparison to commercial medical electrodes in regards of TENS, ECG, and impedance.

Application for Transcutaneous Electrical Nerve Stimulation (TENS). A typical test configuration for TENS application is shown in Fig. 3. In comparison to commercial electrodes, our PHB $+3 \mathrm{wt} \%$ AgNW gel interface electrodes, gave the same thresholds for nerve stimulation using the same stimulation conditions. A test person felt the same level of stimulation with our prototype electrodes as with the commercial electrodes provided with the TENS apparatus. Our developed material is thus a promising alternative to the hard, metallic parts in conventional commercial electrodes.

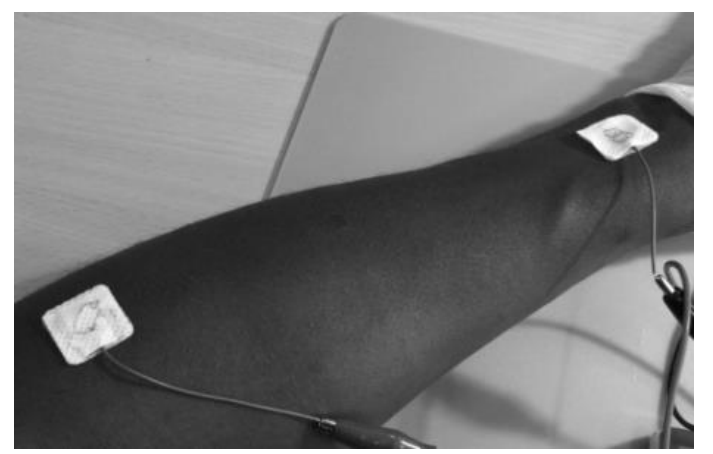

Fig.3: PHB 3\%wt AgNW nanocomposite electrode prototypes in TENS application tests.

Application for Electrocardiography (ECG). We recorded ECG signals using our nanocomposite based electrodes as well as two types of commercial electrodes under the same conditions as typically used for patients. Representative examples of recorded signals are presented in Fig. 4.
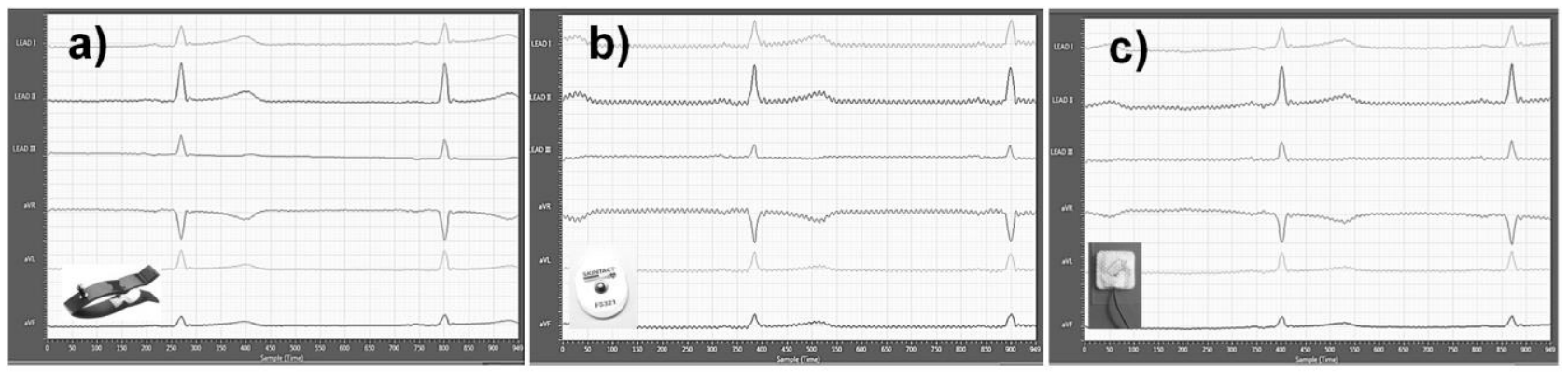

Fig.4: Comparison of ECG acquisition signals using different electrodes: a) system electrode of the ECG system; b) commercial Skintact FS321 electrode; c) PHB + $3 \mathrm{wt} \%$ AgNW prototype electrode. Signals from top to bottom: channels (1) to (3) pairwise potential differences between left hand (LH), right hand (RH) and left leg (LL); channels (4) to (6) pairwise potential differences between right hand, left hand, and left leg with reference points. 
Comparison of the ECG signals recorded using our nanocomposite electrodes (Fig. 4c) with those recorded using the provided system electrodes and commercial skin contact electrodes (Fig. 4a and Fig. 4b, respectively), show that our nanocomposite electrodes allow the ECG signal acquisition without any difficulty and well reproduce the signal shapes. In particular, the level of the signal noise of our $3 \mathrm{wt} \%$ PHB nanocomposite electrode (Fig. 4c) is lower than the one of the studied commercial skin contact electrode (Fig. 4b).

Impedance of prototype electrodes. Since the impedance as a function of frequency is a key parameter for medical electrodes, in particular for ECG applications, for which the signal shape would be affected in case of significant frequency dependencies of the impedance and phase, we measured the impedance and phase of our developed PHB $+3 \mathrm{wt} \%$ AgNW electrodes. Fig. 5 displays for the frequency range of 1 to $100 \mathrm{~Hz}$ an impedance of $(62.3 \pm 1.2) \mathrm{k} \Omega$ with a rather constant phase (between 0.50 and $0.60 \mathrm{rad}$ up to $50 \mathrm{~Hz}$; between 0.45 and $0.50 \mathrm{rad}$ above $50 \mathrm{~Hz}$ ). This behavior confirms the suitability of the nanocomposite for the acquisition of electrical signals such as ECG.
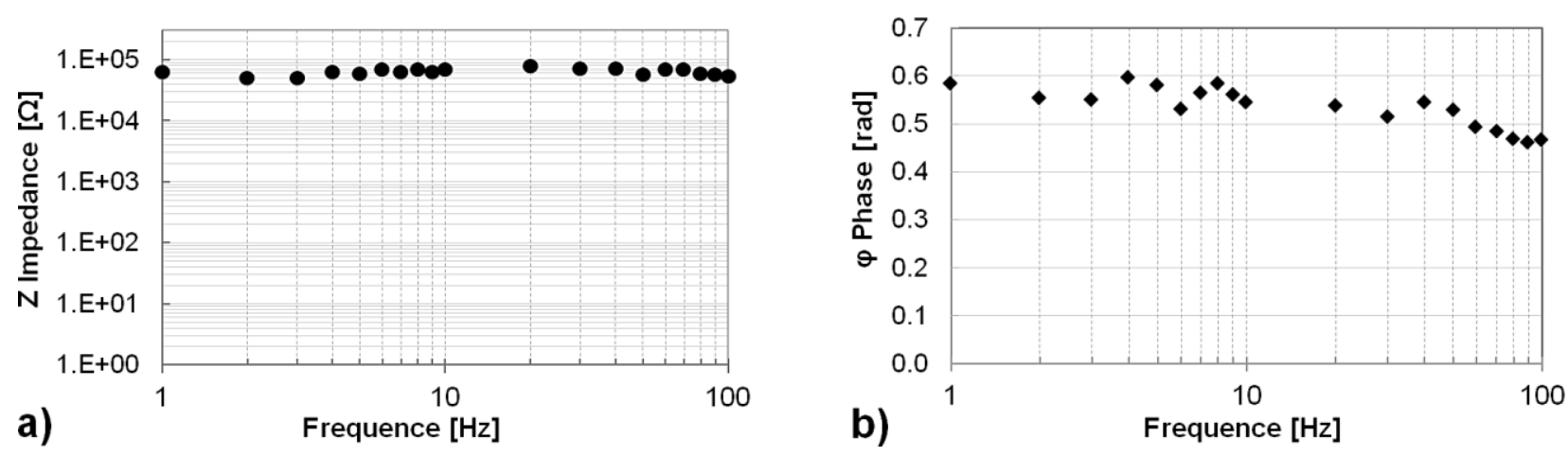

Fig.5: Impedance spectra of the nanocomposite based electrode (PHB + 3wt $\%$ AgNW): a) impedance, b) phase.

\section{Conclusion}

In summary, novel prototype medical electrodes based on nanocomposite biopolymers have successfully been assembled and characterized.

Our study of the electrical conductivity and the microstructure of nanocomposite films of PHB polymer as a function of AgNW load concentrations, exhibits a microstructural transition of the studied PHB based nanocomposites that correlates with the electrical conductivity. In particular, at low nanowire load concentrations, i.e. at only $3 \mathrm{wt} \%$ silver nanowire content a conductive microporous scaffold nanocomposite structure with dense AgNW packing in the scaffold walls, leading to a conductivity of $(2.53 \pm 0.02) \cdot 10^{2} \mathrm{~S} / \mathrm{m}$, was detected.

Our novel prototypes of medical electrodes using the biopolymer nanocomposites (PHB $+3 \mathrm{wt} \%$ $\mathrm{AgNW}$ ) show promising results in terms of conductivity, signal shape and noise levels observed in TENS and ECG tests in comparison to commercial electrodes. Impedance and phase spectra further underline the suitability of the PHB based nanocomposite for ECG signal acquisition.

\section{Acknowledgements}

This work was financially supported by the project BIOFLEX (HES-SO) under grant number HES-SO/I2_13-03. We kindly acknowledge Hervé Girard, Systems Engineering, HES-SO Valais, for performing SEM measurements.

\section{References}

[1] G.Q. Chen and Q. Wu, The application of polyhydroxyalkanoates as tissue engineering materials, Biomaterials 26 (2005) 6565-6578. 
[2] K. Sudesh, H. Abe, and Y. Doi, Synthesis, structure and properties of polyhydroxyalkanoates: biological polyesters. Prog. Polym. Sci. 25 (2000) 1503-1555.

[3] P. L. B. Araujo, C. R. P. C. Ferreira, and E. S. Araujo, Biodegradable conductive composites of poly(3-hydroxybutyrate) and polyaniline nanofibers: Preparation, characterization and radiolytic effects, eXPRESS Polymer Letters 5 (2011) 12-22.

[4] Hong Lu, Samy A. Madbouly, James A. Schrader, Micheal R. Kessler, David Grewell, and William R. Graves. Novel bio-based composites of polyhydroxyalkanoate (PHA)/distillers dried grains with solubles (DDGS), RSC Adv. 4 (2014) 39802.

[5] Thibault Gerard and Tatiana Budtova, Morphology and molten-state rheology of polylactide and polyhydroxyalkanoate blends, Eur. Polym. J. 48 (2012) 1110-1117.

[6] Mònica Bassas-Galià, Adolfo Gonzalez, Fabrice Micaux, Vanessa Gaillard, Umberto Piantini, Silvia Schintke, Manfred Zinn, and Marc Mathieu, Chemical Modification of Polyhydroxyalkanoates (PHAs) for the Preparation of Hybrid Biomaterials, Chimia 69 (2015) 627-629.

[7] Mega Soft Patient Return Electrode for use during monopolar electrosurgery. National Institute for Health and Clinical Excellence, 2012. NICE medical technology guidance [MTG11], ISBN 9781-4731-1177-6, https://www.nice.org.uk/guidance/mtg11.

[8] Shengzhe Yang, Samy A. Madbouly, James A. Schrader, David Grewell, Michael R. Kessler, and William R. Graves, Processing and characterization of bio-based poly (hydroxyalkanoate)/poly(amide) blends: Improved flexibility and impact resistance of PHA-based plastics, J. Appl. Polym. Sci. 132, (2015) 42209.

[9] S. Philip, T. Keshavarz, and I. Roy, Polyhydroxyalkanoates: biodegradable polymers with a range of applications, J. Chem. Technol. Biotechnol. 82 (2007) 233-247.

[10] M. Zinn, H.-U. Weilenmann, R. Hany, M. Schmid, and Th. Egli, Tailored synthesis of poly([R]3-hydroxybutyrate-co-3-hydroxyvalerate) (PHB/HV) in Ralstonia eutropha DSM 428, Acta Biotechnol. 23 (2003) 309-316.

[11] Dieter K. Schroder, Semiconductor material and device characterization. John Wiley \& Sons, Inc. 2006, ISBN 978-0-471-73906-7. 